\title{
Lumbosciatalgia Due to Pyramidal Syndrome
}

\author{
Maria Jose Garcia-Antelo ${ }^{\mathrm{a}, \mathrm{c}}$, Eduardo Rubio-Nazabal ${ }^{\mathrm{a}}$, Adrian Lopez-Baz ${ }^{\mathrm{a}}$, \\ Cristina Mendez-Diaz
}

\begin{abstract}
Piriformis syndrome is a poorly known cause of sciatica and there are few cases reported in the international literature where sciatica is the form of presentation. It is caused by compression or entrapment of the sciatic nerve due to piriformis muscle pathology. A 65-year-old Caucasian man was admitted to the Department of Neurology because he suffered from sciatica and weakness of the left lower limb, so initially a disc herniation was suspected. Upon physical examination, he presented marked gluteal muscle atrophy, mild quadriceps muscle atrophy and paresis of the left lower extremity with distal dominance. Electroneuromyography provided evidence of left sciatic nerve injury of severe intensity and profuse signs of active denervation present. MRI lumbar plexus revealed asymmetrical enlargement of the left piriformis muscle that obliterated the ipsilateral greater sciatic foramen, compressing the left sciatic nerve. As a result, secondary atrophy of the gluteus maximus and medius can be observed on this side. Due to the severity of the symptoms, surgery was performed to release the nerve. Piriformis syndrome rarely occurs as sciatica. The diagnosis is usually delayed because of the absence of further pathognomic testing and established diagnostic criteria, which are based on clinical manifestations. This syndrome is often confused with lumbar-sacral or hip disease. So, in a disease such as the case presented, the piriformis syndrome should be considered. We think that it is very interesting to know that a piriformis syndrome can debut as a lumbosciatalgia and it can be confused with lumbar disc pathology, so this may delay the diagnosis and cause further suffering to the patient.
\end{abstract}

Keywords: Piriformis syndrome; Sciatica; Sciatic nerve; Piriformis muscle; Weakness; MRI

\section{Introduction}

Piriformis syndrome (or pyramidal syndrome) is an entrapment

Manuscript accepted for publication May 04, 2016

aDepartment of Neurology, Complexo Hospitalario Universitario A Coruna, A Coruna, Spain

bDepartment of Radiology, Complexo Hospitalario Universitario A Coruna, A Coruna, Spain

${ }^{\mathrm{c} C o r r e s p o n d i n g ~ A u t h o r: ~ M a r i a ~ J o s e ~ G a r c i a-A n t e l o, ~ D e p a r t m e n t ~ o f ~ N e u r o l o g y, ~}$ Complexo Hospitalario Universitario A Coruna, C/As Xubias, SN, 15006, A Coruna, Spain. Email: marigaran@gmail.com

doi: http://dx.doi.org/10.14740/jmc2503w neuropathy caused by compression of the sciatic nerve involving pathology of the overlying piriformis muscle. There are many entities that can cause piriformis syndrome [1], such as hypertrophy or shortening of the pyramidal muscle, post-traumatic fibrosis, myositis ossificans, total hip arthroplasty, intense physical activity, etc. Its clinical presentation is characterized by pain in the gluteal region that can radiate to the posterior portion of the thigh associated with sensory, motor and trophic impairment in its area of innervation. The clinical manifestations do not have to be ongoing. They can be revealed with certain maneuvers or with prolonged sitting. On very rare occasions, it is associated with lumbar pain and simulates lumbosciatalgia. Complementary examinations usually show no abnormalities, therefore, piriformis syndrome is commonly confused with other types of diseases making it often under-diagnosed. We present the case of a patient with sciatalgia related to severe piriformis syndrome.

\section{Case Report}

A 65-year-old male with hypertension and diabetes who was undergoing treatment with amlodipine and glibenclamide, without other notable medical or surgical history, was hospitalized for clinical signs and symptoms of lumbosciatalgia with weakness and atrophy of the left lower extremity that had progressed over 2 months. Upon physical examination (Fig. 1), he presented marked gluteal muscle atrophy and mild quadriceps muscle atrophy of the left lower extremity; inability to dorsiflex the left foot $(0 / 5)$ and difficulty in plantar flexion $(3 / 5)$ as well as proximal weakness (4/5). In addition, he presented tacto-algesic hypoesthesia in the left L5-S1 territory and left steppage gait. The rest of the physical examination was normal. Suspecting herniated disc, a lumbar magnetic resonance imaging (MRI) was performed, which showed no abnormalities. To complete the study, blood tests were carried out and were normal (complete blood count, biochemistry, thyroid-stimulating hormone (TSH), folic acid, B12, C-reactive protein (CRP), antinuclear antibodies (ANAs) and anti-neutrophil cytoplasmic antibodies (ANCAs), hepatitis, lues and human immunodeficiency virus (HIV) serology, proteinogram, and onconeuronal antibodies); the cerebral spinal fluid showed no abnormalities. Electroneuromyography provided evidence of left sciatic nerve injury of severe intensity and profuse signs of active denervation present. MRI lumbar plexus (Figs. 2 and 3) revealed asymmetrical enlargement of the left piriformis muscle that obliterated the ipsilateral greater sciatic foramen, compressing the left sciatic nerve. As a result, secondary atrophy of the gluteus maximus and medius was observed on this side. The diagnosis of left sciatalgia due to py- 


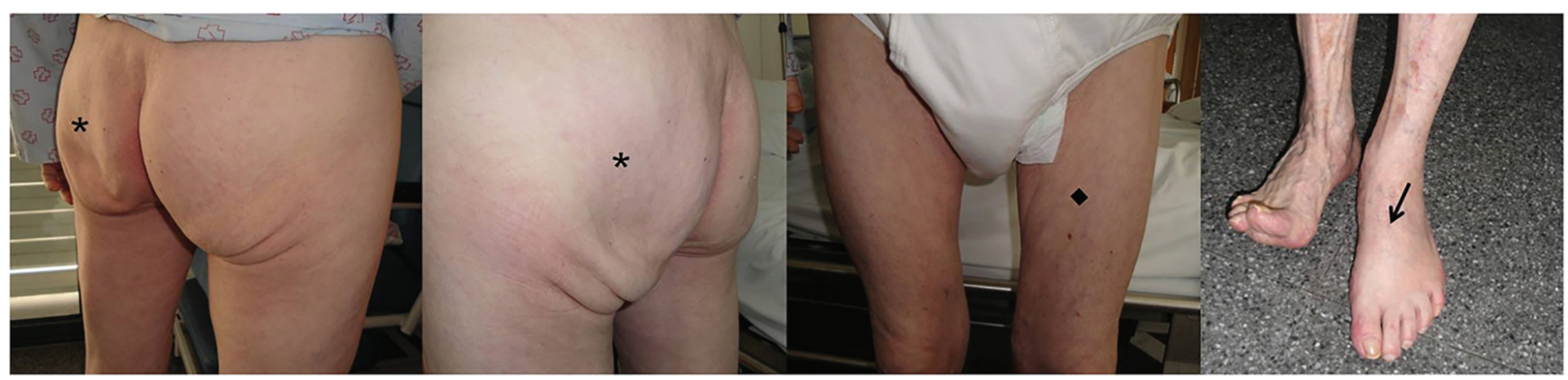

Figure 1. Photograph of the lower extremities of the patient. $\left({ }^{*}\right)$ Marked left gluteal muscle atrophy. $(\star)$ Mild left quadriceps muscle atrophy. $(\rightarrow)$ Inability to dorsiflex the left foot $(0 / 5)$.

ramidal syndrome was made related to pyramidal muscle hypertrophy, of unknown etiology. Due to the severity of the clinical picture, surgery was performed, consisting of the dissection of the left sciatic nerve along its entire tract from the distal third of the left thigh to the piriformis muscle and the section of the annulus fibrosus located at the greater sciatic notch. A partial disinsertion of the left gluteus medius and piriformis muscles was also performed at the level of the greater trochanter of the femur. He subsequently received rehabilitation treatment, presenting partial clinical improvement in strength and muscle mass.

\section{Discussion}

Piriformis syndrome is an entrapment neuropathy of a highly variable nature in which the sciatic nerve is compromised by hypertrophy or contracture of the piriformis muscle. The typical clinical manifestation is usually in the form of deep pain in the region of the lower third of the gluteus, radiating to the lower extremity. In general, it does not involve constant pain, but can be triggered by certain activities such as prolonged sitting, climbing up and down stairs, etc. However, in a percentage of patients, the pain is in the lumbar region, radiating to the ipsilateral lower extremity as if it were lumbosciatalgia that could make us suspect disc disease at this level. In addition to pain, which is the most common clinical manifestation, other rare signs and symptoms could appear in cases where nerve compromise is more severe. There may be tingling or numbness in the leg, atrophy of the musculature dependent upon the sciatic nerve and paresis thereof.

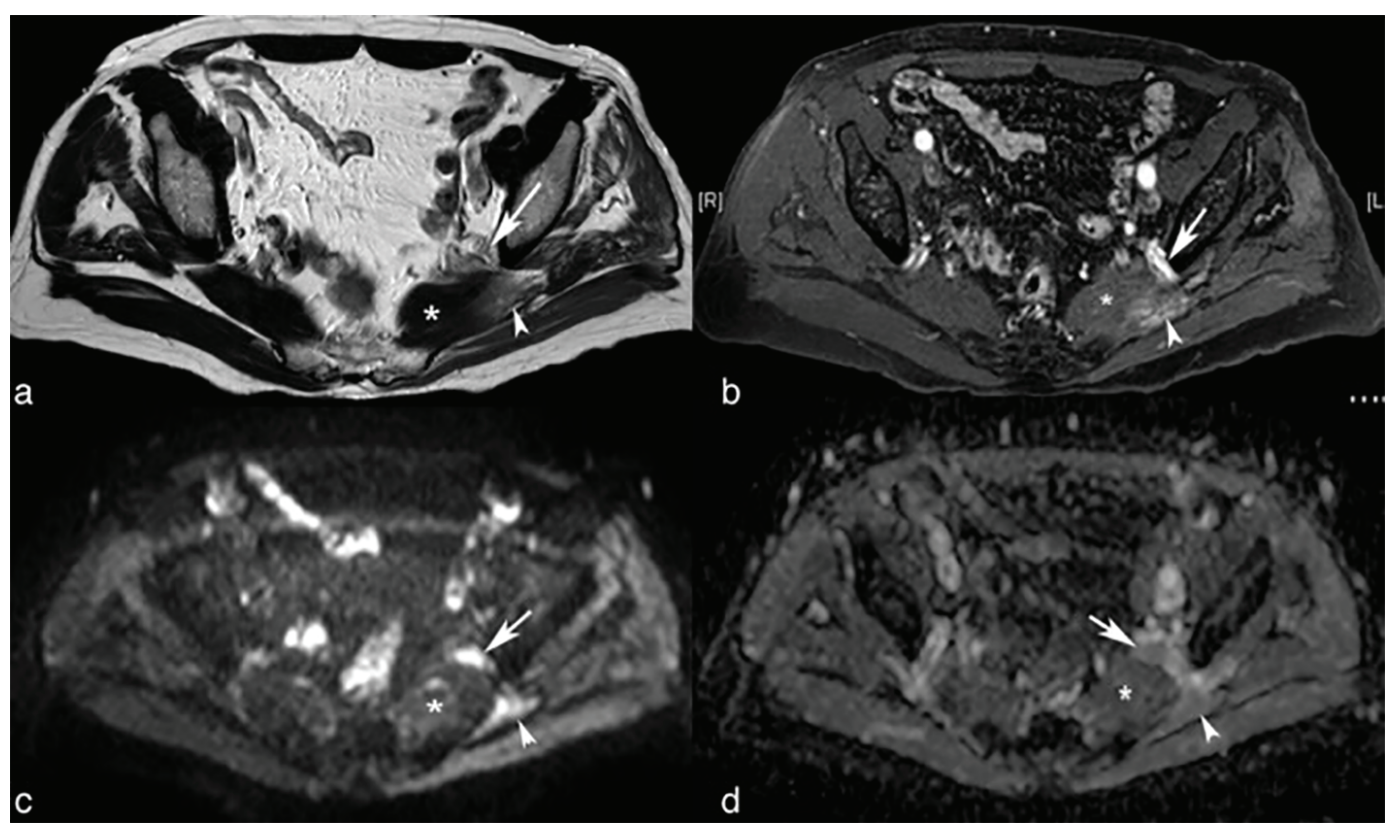

Figure 2. MRI imaging. Axial TSE-T2-weighted (a), gadolinium enhanced fat-suppressed T1-weighted (b), b 1000 diffusionweighted image (c) and apparent diffusion coefficient map (d) images show a hypertrophied left piriformis muscle (asterisks) compressing the left sciatic nerve (arrow). The left sciatic nerve (arrows) shows increased size and signal intensity on T2weighted image, enhancement after gadolinium administration and restricted diffusion. Subacute denervation changes on mulateral portion of the hypertrophied left piriformis (arrowheads) and gluteus medius muscles and chronic denervation changes on gluteus minimus muscle also can be seen. 


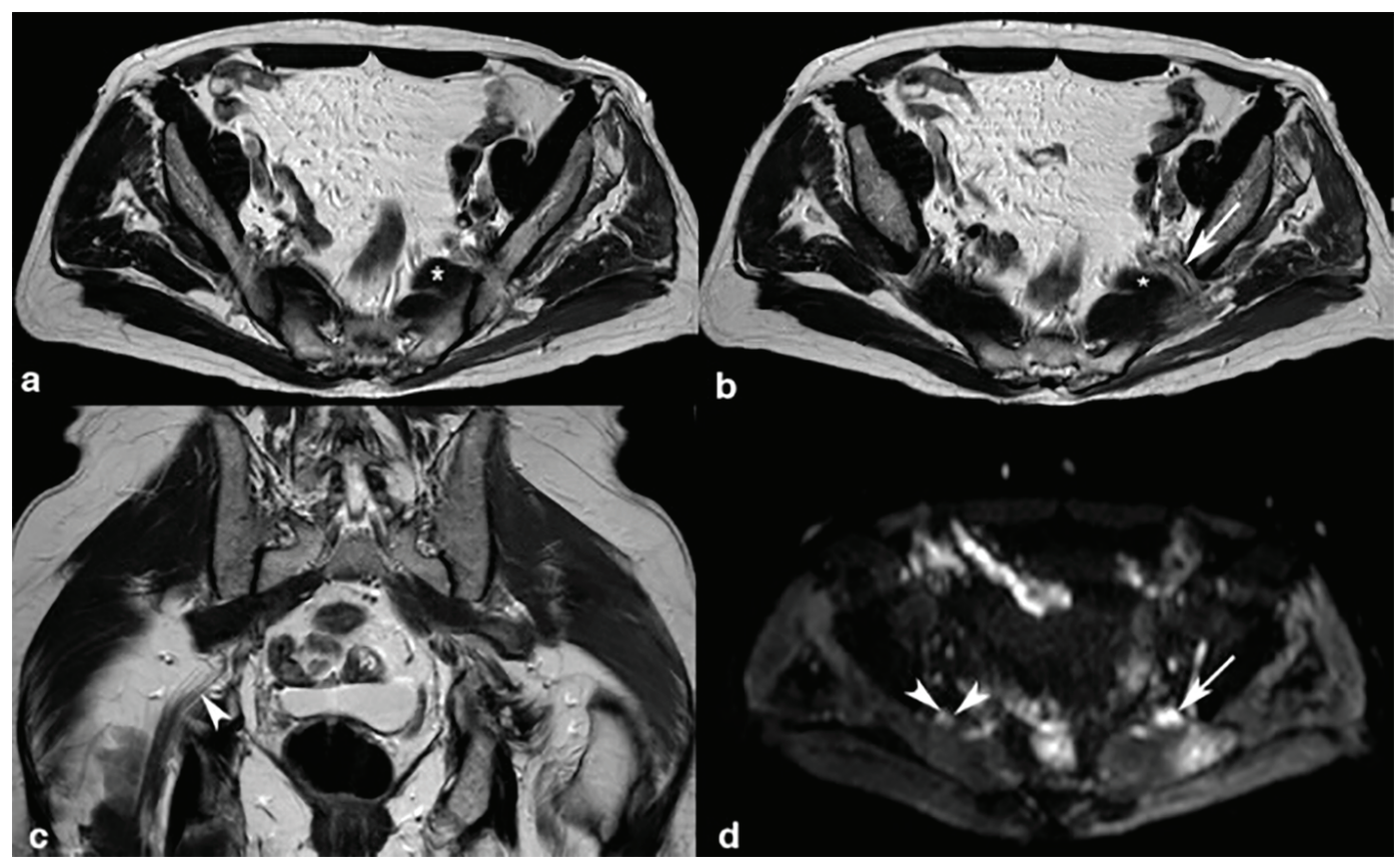

Figure 3. MRI imaging. Axial (a, b) and coronal (c) TSE-T2-weighted images show accessory fibers (asterisks) of left piriformis muscle compressing a thickened left sciatic nerve (arrow). The enlarged left sciatic nerve (arrows) shows increase signal intensity on T2-weighted image and on b 1000 diffusion-weighted image (d). Note the fascicular composition, perifascicular fat and signal intensity of normal right sciatic nerve (arrowheads). Subacute denervation changes on lateral portion of the hypertrophied left piriformis owing to involvement of the sacral plexus also can be seen.

For this reason, it is critical to perform careful physical examination to guide us to the location of the injury. The following signs are indicative of piriformis syndrome [2]: 1) Lasegue's test: negative. 2) Piriformis sign: the patient is in the supine position with the legs straight. The leg that has piriformis syndrome presents external rotation compared to the other. 3) Freiberg's sign: the patient is in the supine position with the lower extremities in extension. Forced passive internal hip rotation reproduces the pain by stretching the piriformis muscle. 4) Pace sign: the patient is sitting. Pain is present on performing external hip rotation and abduction. If the pain is localized in the lower part of the gluteus, it indicates piriformis syndrome. If the pain occurs in the lumbar region, radiating to the leg, it indicates disc disease. 5) Beatty test: the patient is lying on the healthy side with the affected hip and the knee flexed. Lift and hold the superior knee approximately a few centimeters from the examination table. If this reproduces sciatica symptoms, the result is positive and indicates piriformis injury. 6) FAIR test: the patient is in a lateral recumbent position, lying on the healthy side. The affected hip is flexed to $60^{\circ}$. The affected knee bent to $60-90^{\circ}$. The hip is stabilized. The examiner internally rotates the hip and uses the approach of applying downward pressure on the knee. The test is positive if the symptoms reoccur, therefore indicating piriformis injury.

The reason that this occurs is not well defined, but it seems to be related to chronic injury to the piriformis muscle, which contracts leading to edema, and as a result, sciatic nerve entrapment. The main causes are both traumatic (post-traumatic fibrosis of the pyramidal muscle, myositis ossificans, total hip arthroplasty) and muscle strain (spending long periods sitting) or after intense physical activity (marathon runners, walkers), etc. The diagnosis is usually delayed because of the absence of further pathognomic testing and established diagnostic criteria [3]; so, it is based on clinical manifestations, which are often confused with lumbar-sacral or hip diseases. It can be confused with discitis, with lumbosacral plexopathy, sacroiliitis, sciatica and trochanteritis or bursitis. Except in very advanced cases, the electroneuromyography will be normal. The MRI lumbar plexus sometimes shows sciatic nerve inflammation [4]. Therefore, exploratory maneuvers are essential in helping us to make the diagnosis.

In the majority of cases, conservative treatment can be established with non-steroidal anti-inflammatory (NSAIDs), corticoid injections or anesthetic agents and botulinum toxin. Rarely and only in severe cases, as described above, surgery is needed [5].

\section{Conclusion}

Piriformis syndrome rarely occurs as sciatica. The diagnosis is usually delayed because of the absence of further pathognomic testing and established diagnostic criteria, which are based on clinical manifestations. This syndrome is often confused with lumbar-sacral or hip disease. So, in a patient with pain in the gluteal region, sometimes radiating to the lower extremity, with poor tolerance to sitting or in cases of lumbosciatalgia without significant abnormalities on the lumbar MRI, this syndrome should be considered. Moreover, we think that it is 
very interesting to know that a piriformis syndrome can debut as a lumbosciatalgia and it can be confused with lumbar disc pathology, so this may delay the diagnosis and cause further suffering to the patient.

\section{Grant}

We have not had any kind of subsidy for this work.

\section{Conflicts of Interest}

The authors declare that they have no competing interests

\section{Abbreviations}

ANAs: antinuclear antibodies; ANCAs: anti-neutrophil cytoplasmic antibodies; CRP: C-reactive protein; HIV: human immunodeficiency virus; MRI: magnetic resonance imaging; NSAIDs: non-steroidal anti-inflammatory; TSH: thyroid-stim- ulating hormone

\section{References}

1. Boyajian-O'Neill LA, McClain RL, Coleman MK, Thomas PP. Diagnosis and management of piriformis syndrome: an osteopathic approach. J Am Osteopath Assoc. 2008;108(11):657-664.

2. Kirschner JS, Foye PM, Cole JL. Piriformis syndrome, diagnosis and treatment. Muscle Nerve. 2009;40(1):1018.

3. Michel F, Decavel P, Toussirot E, Tatu L, Aleton E, Monnier G, Garbuio P, et al. The piriformis muscle syndrome: an exploration of anatomical context, pathophysiological hypotheses and diagnostic criteria. Ann Phys Rehabil Med. 2013;56(4):300-311.

4. Lee EY, Margherita AJ, Gierada DS, Narra VR. MRI of piriformis syndrome. AJR Am J Roentgenol. 2004;183(1):63-64.

5. Indrekvam K, Sudmann E. Piriformis muscle syndrome in 19 patients treated by tenotomy - a 1- to 16-year follow-up study. Int Orthop. 2002;26(2):101-103. 